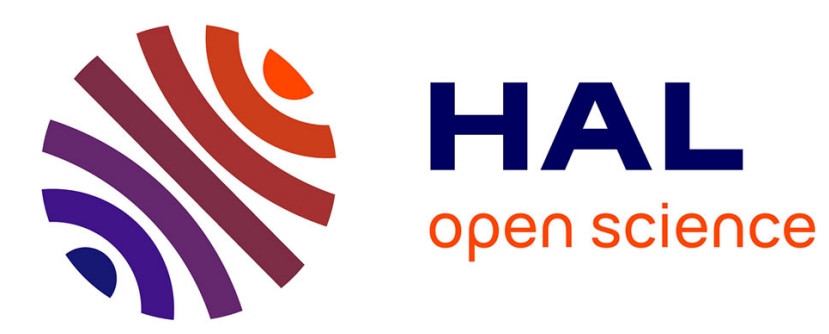

\title{
A boundary integral equation method for the calculation of the effective permittivity of periodic composites
}

Bruno Sareni, Laurent Krähenbühl, Abderrahmane Beroual, Alain Nicolas, C. Brosseau

\section{- To cite this version:}

Bruno Sareni, Laurent Krähenbühl, Abderrahmane Beroual, Alain Nicolas, C. Brosseau. A boundary integral equation method for the calculation of the effective permittivity of periodic composites. IEEE Transactions on Magnetics, 1997, 33 (2 Part 2), pp.1580-1583. hal-00140678

\section{HAL Id: hal-00140678 https://hal.science/hal-00140678}

Submitted on 23 Apr 2007

HAL is a multi-disciplinary open access archive for the deposit and dissemination of scientific research documents, whether they are published or not. The documents may come from teaching and research institutions in France or abroad, or from public or private research centers.
L'archive ouverte pluridisciplinaire HAL, est destinée au dépôt et à la diffusion de documents scientifiques de niveau recherche, publiés ou non, émanant des établissements d'enseignement et de recherche français ou étrangers, des laboratoires publics ou privés. 


\title{
A Boundary Integral Equation Method for the Calculation of the Effective Permittivity of Periodic Composites
}

\author{
B. Sareni, L. Krähenbühl, A. Beroual, A. Nicolas \\ Ecole Centrale de Lyon, CEGELY, UPRESA C.N.R.S. 5005, BP 163, 69131 Ecully Cedex - France: \\ C. Brosseau \\ Laboratoire d'Etude des Matériaux, Université de Bretagne Occidentale, BP 809, 29285 Brest Cedex - France.
}

\begin{abstract}
We present a numerical method based upon the resolution of boundary integral equations for the calculation of the effective permittivity of a lossless composite structure consisting of a two component mixture, each with its own dielectric and shape characteristics. The topological arrangements considered are periodic lattices of inhomogeneities. Our numerical simulations are compared to the effective medium approach and with results of previous works.
\end{abstract}

\section{INTRODUCTION}

In recent years industries such as the aerospace, electronics, and telecommunications have continuously supported the development of new materials and particularly composites for their capability to improve sensitivity and selectivity of devices. A wide variety of applications have been studied e.g. captive video disk units, electromagnetic absorbing materials, microwave systems, carbon-black polymer to cite but a few. The characterization of heterogeneous media requires a rigorous topological analysis. The dielectric properties of these structures strongly depend on the internal structure of the medium i.e. the shape, the volume fractions, and the arrangement of the different components. At first, this paper describes the effective medium approach for the calculation of the dielectric constant of composite materials. We further present the application of boundary integral equation method and discuss the obtained results for various shape of the components. Finally, we show how the dielectric properties of the composite can be affected by the topological arrangement of inhomogeneities especially for crystalline lattices.

\section{EFfECTIVe Medium ApPROACH}

In low frequency modeling of composite materials, the concept of effective permittivity can be used to describe media that are quasi homogeneous to the extent that no scattering effects are significant as the wave propagates into these materials. The spatial variation of the incident electric field must be very large compared to the size of the inhomogeneities dispersed in the medium. The limit for the size of the inclusions is about $\lambda / 2 \pi$ where $\lambda$ is the wavelength of the electric field probing the medium [1]. At higher frequencies scattering effects take over and the concept of effective permittivity loses its meaning. Consider an inhomogeneous mixture consisting of inclusions with permittivity $\varepsilon_{1}$ embedded in a host medium of permittivity $\varepsilon_{2}$. The average displacement vector $\bar{D}$ is related to the macroscopic field $\bar{E}$ as follows

$$
\bar{D}=\varepsilon \bar{E}=\varepsilon_{2} \bar{E}+\bar{P}
$$

where $\bar{P}$ is the polarization in the material.

The polarization can be calculated from the effective or local electric field $\bar{E}_{e}$ :

$$
\bar{P}=N \alpha \bar{E}_{i}
$$

$N$ being the number of polarizable inhomogeneities per unit volume, $\alpha$ being the polarisability of the inclusions.

The effective electric field is the sum of the macroscopic field $\vec{E}$ and the depolarization field which is calculated using the decomposition of Yaghjian [2]:

$$
\bar{E}_{\psi}=\bar{E}+\frac{1}{\varepsilon_{2}} \overline{\bar{L}} \cdot \bar{P}
$$

where the source dyadic $\overline{\bar{L}}$ depends on the shape of the inclusions. From the previous equations, one can express the effective permittivity of the medium as follows:

$$
\varepsilon=\varepsilon_{2}+N \alpha\left(1-\frac{1}{\varepsilon_{2}} \overline{\bar{L}} \cdot N \alpha\right)^{-1}
$$

Equation (4) requires the calculation of the polarizability $\alpha$. This entails solving the internal and external fields of an inclusion i.e. to find analytical solution to the Laplace's equation. Approximate solutions can be found for particular shapes of the inhomogeneities e.g. spherical, ellipsoidal, or cylindrical particles.

Unfortunately this approach cannot take into account the interaction effects between scatterers and therefore is limited to dilute mixtures. Moreover, the case of touching inhomogeneities cannot be considered because of problems associated to boundary conditions.

\section{BOUNDARY INTEGRAL EQUATION METHOD}

The solution of the Laplace equation can be computed by applying boundary integral equation (BIE) method. Upon using Green's theorem, we can write the local potential $V(M)$, inside a spatial region $\Omega$, in terms of $V(P)$, and the normal derivative $\partial V(P) / \partial n, P$ being any point on the boundary $\Sigma$ (with no overhangs) of $\Omega$ :

$V(M)=-\frac{4 \pi}{A} \int_{\Sigma}\left(V(P) \frac{\partial G(P, M)}{\partial n}-G(M, P) \frac{\partial V(P)}{\partial n}\right) d s$

where $A$ denotes the solid angle under which the point $M$ sees the oriented surface $\Sigma, n$ is the normal unit vector 
oriented outward to $\Sigma, d s$ is a surface element of $\Sigma$ and $G$ is the Green function defined in [3].

Considering the property of periodicity of the structure, the composite geometry is reduced to a unit cell. The constituent of permittivity $\varepsilon_{1}$ occupying the volume $\Omega_{1}$ is embedded in the region $\Omega_{2}$ of permittivity $\varepsilon_{2}$. In that case (5) reads for the domain $i(i=1,2)$ as

$$
V=-\frac{4 \pi}{A} \int_{\Sigma_{i}}\left(V \frac{\partial G}{\partial n}-\left.G \frac{\partial V}{\partial n}\right|_{i}\right) d s
$$

Moreover, we have

$$
\left.\varepsilon_{1} \frac{\partial V}{\partial n}\right|_{1}=\left.\varepsilon_{2} \frac{\partial V}{\partial n}\right|_{2}
$$

by the virtue of interface conditions. The implementation of the BIE method consists in dividing the boundaries into finite elements and for each finite element, the potential and its normal derivative are calculated on the nodes thanks to interpolating functions [3]. Following this way, integral equations are transformed into a matrix equation which is solved, from the boundary conditions displayed in Fig. $I$ and 2, using numerical technique e.g. Gauss method.

Hence, the effective permittivity of the medium is obtained from the potential and its normal derivative by considering two types of configuration:

I) Inclusions are isolated (sparse mixture). The medium of permittivity $\varepsilon_{1}$ cannot intercept the sides of the parallelipipedic cell (see Fig. 1). In that case, the effective permittivity in the direction corresponding to the applied field is calculated using the following relation:

$$
\left.\int_{S} \varepsilon_{2} \frac{\partial V}{\partial n}\right|_{2} d s=\varepsilon_{z} \frac{V_{2}-V_{1}}{e} S
$$

where $V_{2}-V_{1}$ denotes the slope of potential imposed in the $z$ direction, $e$ the thickness in the same direction, and $S$ the side surface of the unit cell perpendicular to the applied field.

2) Inclusions are allowed to fused each other (dense mixture). The region of permittivity $\varepsilon_{1}$ can intercept the sides of the unit cell (see Fig. 2). In that case, we have to take into account the electric displacement flux through the area $S_{1}$ for the calculation of the effective permittivity in the direction corresponding to the applied field:

$$
\left.\int_{S_{2}} \varepsilon_{2} \frac{\partial V}{\partial n}\right|_{2} d s+\left.\int_{S_{1}} \varepsilon_{1} \frac{\partial V}{\partial n}\right|_{1} d s=\varepsilon_{z} \frac{V_{2}-V_{1}}{e}\left(S_{1}+S_{2}\right)
$$

In these two configurations, the effective permittivity in the other directions ( $x$ and $y$ ) can be found similarly by modifying the boundary conditions in the unit cell.

\section{RESULTS}

We turn now to a presentation of our numerical results concerning the effective permittivity of a composite as a function of the permittivities and the volume fraction of the

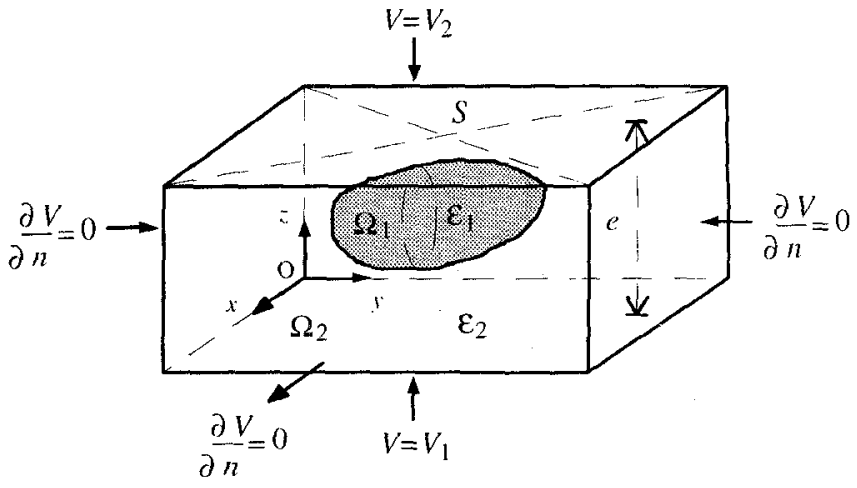

Fig. 1. Boundary conditions in the unit cell of a sparse composite.

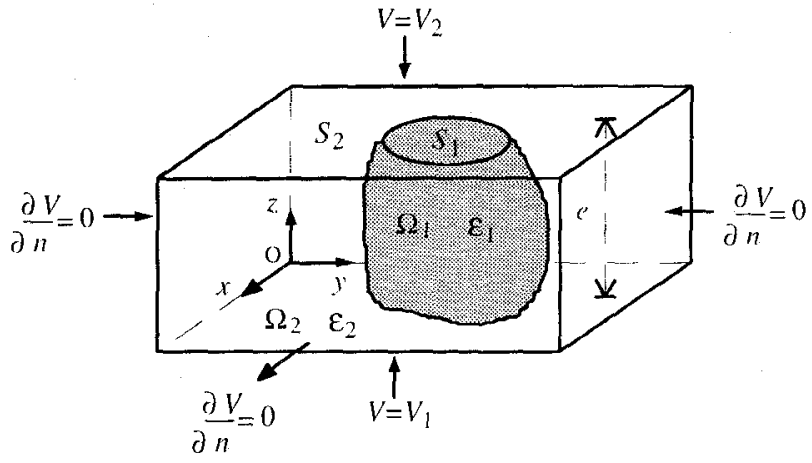

Fig. 2. Boundary conditions in the unit cell of a dense composite.

constituents. In the subsequent sections, we consider different shapes of inhomogeneities and compare the data obtained by the BIE method with standard analytical calculations resulting from the effective medium approach. Finally, we examine how the effective permittivity can be affected by the crystalline arrangement of the periodic structure.

\section{A. Composite with spherical inclusions}

Consider a periodic composite with spherical inclusions of permittivity $\varepsilon_{1}$ embedded in a crystalline fashion (simple cubic lattice) in a homogeneous matrix of permittivity $\varepsilon_{2}$. The material can be described by a unit cell consisting in a spherical inclusion of radius $r$ centered in cube of unit side. Beyond the touching spheres condition $(r>0.5)$, the sphere is allowed to grow and intercept spheres in adjacent cells according to [4]. The unit cell structure is described in Fig. 3. The effective permittivity of the medium is calculated from the polarizability of the inhomogeneities

$$
\alpha=4 \pi r^{3} \varepsilon_{2} \frac{\varepsilon_{1}-\varepsilon_{2}}{\varepsilon_{1}+2 \varepsilon_{2}}
$$

and the dyadic source $\overline{\bar{L}}=\bar{I} / 3(\bar{I}$ being the unit tensor) by substituting these values in (4). This leads to Rayleigh formula [5]:

$$
\varepsilon=\varepsilon_{2} \frac{\varepsilon_{1}+2 \varepsilon_{2}+2 f\left(\varepsilon_{1}-\varepsilon_{2}\right)}{\varepsilon_{1}+2 \varepsilon_{2}-f\left(\varepsilon_{1}+2 \varepsilon_{2}\right)}
$$


where $f$ denotes the volume fraction of the inclusions. The values of the effective permittivity resulting from our numerical simulations are compared to those computed by a Fourier expansion technique [4] and deduced from (11). (see Fig. 4). The data obtained by the BIE method and in [4] are quite similar in the entire range of the volume fraction of inhomogeneities except in the region of percolation $(f=0.523)$. The standard analytical model of Rayleigh is limited to low values of the inclusion concentration.

\section{B. Composite with cubic inclusions}

Now, we consider the effective permittivity of composite with cubic inclusions. The geometry of the unit cell consists of a cubic particle of permittivity $\varepsilon_{1}$ centered in a cubic region of permittivity $\varepsilon_{2}$ as described in [6]. Since for cubic inclusions $\overline{\bar{L}}=\overline{\bar{I}} / 3$, the permittivity of the medium can be also approached with Rayleigh formula. The results obtained by the BIE method are shown in Fig. 5. The behaviour of the effective permittivity at high concentration of inhomogeneities is rather different from that observed in Fig. 4. We explain this observation from the fact that, due to the particular geometry, inclusions cannot intercept each other over the entire range of $f$. Therefore, the electric field is always concentrated in the region of low permittivity.

\section{Composite with ellipsoidal inclusions.}

For periodic mixtures of ellipsoidal inclusions, the effective permittivity is calculated by Sillars formula resulting from the effective medium approach [5]. Since ellipsoidal inclusions are spatially dissymmetric, the medium is anisotropic. The effective permittivity is described by a diagonal tensor composed of three components corresponding to the permittivity in the directions $x, y$ and $z$

$$
\varepsilon_{i}=\varepsilon_{2}\left(1+\frac{\left(\varepsilon_{1}-\varepsilon_{2}\right) f}{\varepsilon_{2}+\left(\varepsilon_{1}-\varepsilon_{2}\right)(1-f) n_{i}}\right) ; \quad i=x, y, z
$$

where $f$ is the volume fraction of the ellipsoidal inclusions and $n_{i}$ their depolarization factor in the $i$ direction [5].

For our simulations, we consider an array of prolate spheroids $(b=c=a / 4)$ oriented in the $x$ direction. The numerical data computed by the BIE method are compared to those calculated with (12) (see Fig. 6). The great deviation between these two models, particularly in the $x$ direction, can be explained by the fact that Sillars formula is unable to take into account the local field intensification (edge effect) at the extremity of the ellipsoidal particle and the effect of proximity of the inclusions. The BIE method does not suffer from these disadvantages.

\section{Composite with cylindrical inclusions.}

We examine now the effective permittivity of a composite with cylindrical inclusions.

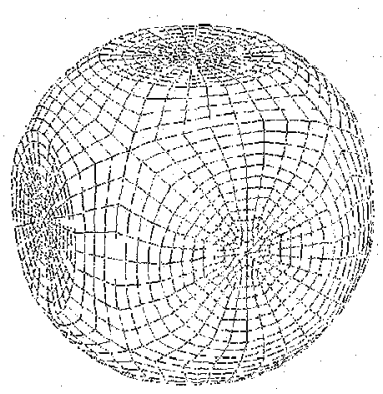

Fig. 3. A mesh of the spherical inclusion in the unit cell of the composite beyond the touching spheres condition.

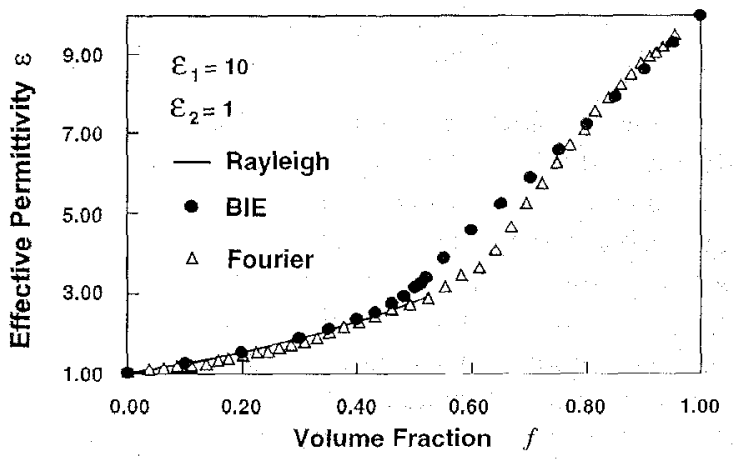

Fig. 4. Effective permittivity of a periodic composite with spherical inclusions.

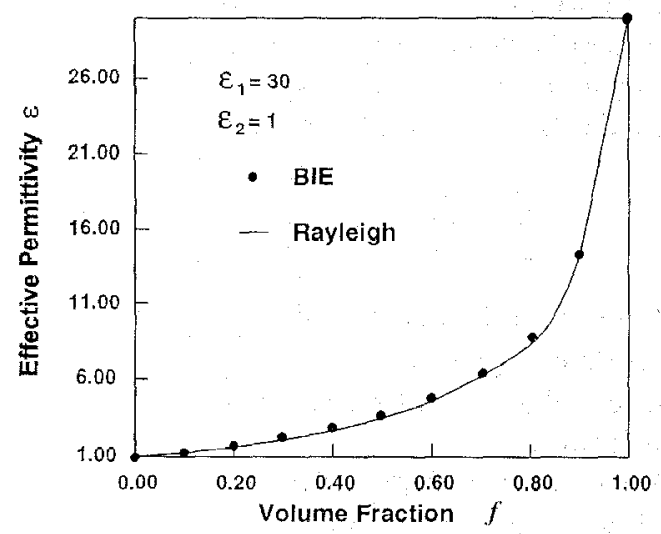

Fig. 5. Effective permittivity of a periodic composite with cubic inclusions.

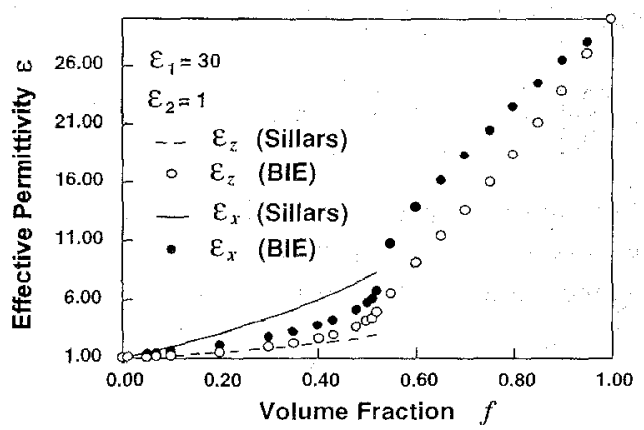

Fig. 6. Effective permittivity of a periodic composite with spheroidal inclusions. 
The unit cell of the material consists in a cylindrical particle of radius $r$, length $h$ and permittivity $\varepsilon_{1}$ embedded in a parallelipipedic region of permittivity $\varepsilon_{2}$. We distinguish between two types of configurations: Inclusions can be assimilated to discs $(r>>h)$ or rods $(r<<h)$. We denote by $\varepsilon_{\perp}$ and $\varepsilon_{/ /}$the effective permittivity in the perpendicular and parallel direction to the cylinder axis respectively. The numerical results obtained by the BIE method for rods $(h=16 r)$ and discs $(r=5 h)$ are displayed in Fig. 7. They illustrate the anisotropy of the medium. It is difficult to compare these data with standard analytical models because they are rigorously valid for infinite cylinders or discs having low thicknesses only [5]. We have noted an important deviation between numerical and analytical models even at low concentration of inhomogeneities.

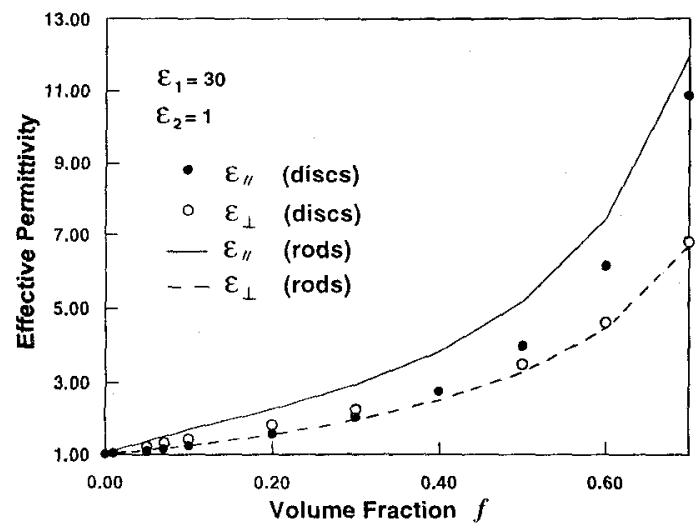

Fig. 7. Effective Permittivity of a periodic composite with cylindrical inclusions.

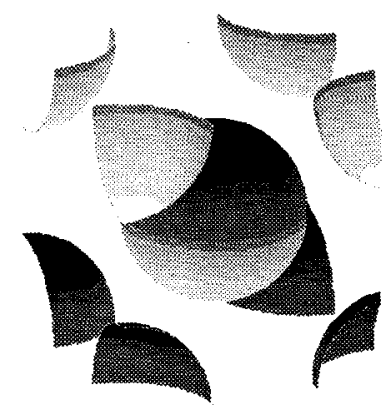

Fig. 8. Field distribution in a body-centered cubic lattice of perfectly conducting spheres.
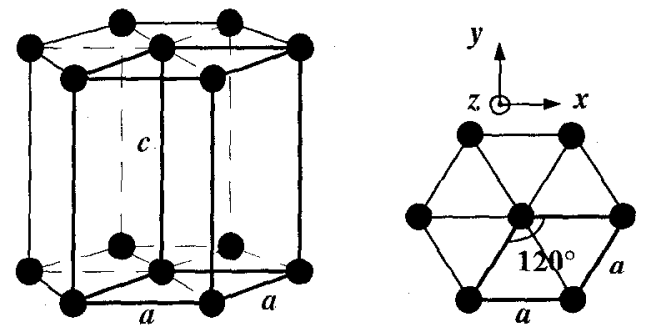

Fig. 9. Internal structure of an hexagonal lattice.

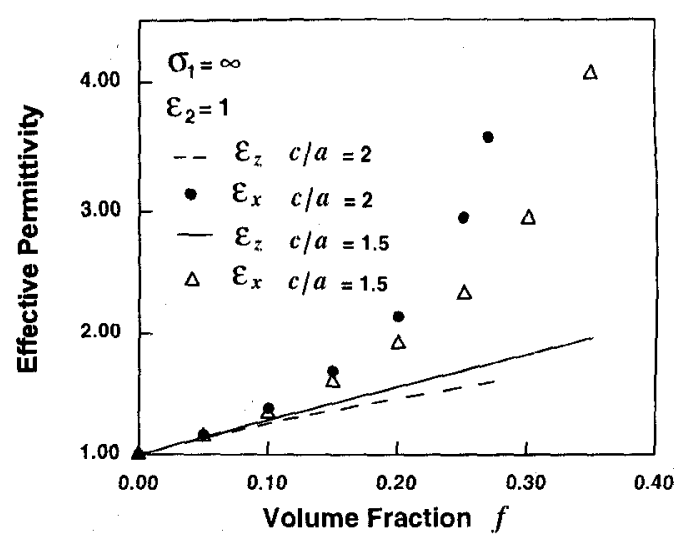

Fig. 10. Effective Permittivity of hexagonal lattices

\section{E. Dependence on the crystalline structure.}

For cubic lattices, results obtained by the BIE method are identical to those presented in [7], [8]. Fig. 8 illustrates the field distribution in a body-centered cubic lattice. Finally, we examine the permittivity of an hexagonal lattice of perfectly conducting spheres $\left(\sigma_{1} \rightarrow+\infty\right)$. The internal structure of the material is displayed in Fig. 9. This type of composite is anisotropic $\left(\varepsilon_{x} \neq \varepsilon_{z}\right)$ and we observe in Fig. 10 that the permittivity depends strongly on the specific ratio cla at low concentration of inhomogeneities.

\section{CONCLUSION}

The BIE method is an efficient and powerful tool to describe the permittivity of lossless periodic composites. It gives accurate results by taking into account edge and proximity effects and is not limited by the volume fraction of inhomogeneities. This numerical technique can be easily extended to multiphase structures containing arbitrarily shaped components.

\section{REFERENCES}

[1] A. Shivola and J.A. Kong, "Effective Permittivity of Dielectric Mixtures," IEEE Trams. on GeoSci. and Remote Sen., vol. 26, no. 4, pp. 420-429, 1988.

[2] A. Yaghjian, "Electric Dyadic Green's Functions in the Source Region," Proc IEEE, vol. 68, no. 2, pp. 248-263,1980.

[3] C.A. Brebbia, The boundary element method for engineers, Pentech Press, London, 1980.

[4] L.C. Shen, C. Liu, J. Korringa, and K.J. Dunn, "Computation of Conductivity and Dielectric Constant of Periodic Porous Media,"J. Appl. Phys., vol. 67, no. 11, pp. 7071-7081, 1990.

[5] L.H.K. Van Beek, "Dielectric Behaviour of Heterogeneous Systems," Progess in Dielectrics, London, vol.7, pp. 69-114, 1967.

[6] P.K. Ghosh and M.E. Azimi, "Numerical Calculation of Effective Permittivity of Losseless Dielectric Mixtures using Boundary Integral Method," IEEE Trans. on Diel. and Elect. Insul., vol. 1, no. 6, pp. 975 $981,1994$.

[7] R.C. McPhedran and D.R. McKenzie,"The Conductivity of Lattices of Spheres. I. The simple cubic lattice," Proc. Roy. Soc.. Ser. A, vol. 359, pp. $45-63,1978$.

[8] W.T. Doyle, "The Clausius-Mossotti Problem for Cubic Array of Spheres,", J. Appl. Phys., vol 49, no. 2, pp. 795-797, 1977. 